\title{
Autoimmune Encephalitis at the Neurological Intensive Care Unit: Etiologies, Reasons for Admission and Survival
}

\author{
Gayane Harutyunyan ${ }^{1}$ Larissa Hauer ${ }^{2} \cdot$ Martin W. Dünser $^{3} \cdot$ Anush Karamyan $^{1}$ • \\ Tobias Moser $^{1} \cdot$ Slaven Pikija $^{1} \cdot$ Markus Leitinger $^{1} \cdot$ Helmut F. Novak $^{1}$ • \\ Eugen Trinka ${ }^{1} \cdot$ Johann Sellner ${ }^{4,5}$
}

Published online: 27 December 2016

(C) The Author(s) 2016. This article is published with open access at Springerlink.com

\begin{abstract}
Background Early recognition and treatment of autoimmune encephalitis (AE) has become an essential issue in clinical practice. However, little is known about patients with deteriorating conditions and the need for intensive care treatment. Here, we aimed to characterize underlying aetiologies, clinical symptoms, reasons for intensive care admission, and mortality of critically ill patients with AE. Methods We conducted a retrospective chart review of all patients with "definite" or "probable" diagnoses of $\mathrm{AE}$ treated at our neurological intensive care unit between 2002 and 2015. We collected and analyzed clinical, paraclinical, laboratory findings and assessed the mortality at last follow-up based on patient records.

Results Twenty-seven patients [median age 55 years (range $25-87)$, male $=16$ ] were included. Thirteen $(48 \%)$ had "definite" AE. The most common reasons for admission were status
\end{abstract}

Electronic supplementary material The online version of this article (doi:10.1007/s12028-016-0370-7) contains supplementary material, which is available to authorized users.

Johann Sellner

j.sellner@salk.at

1 Department of Neurology, Christian Doppler Medical Center, Paracelsus Medical University, Salzburg, Austria

2 Department of Psychiatry, Christian Doppler Medical Center, Paracelsus Medical University, Salzburg, Austria

3 Department of Critical Care, University College of London Hospital, London, UK

4 Department of Neurology, Klinikum rechts der Isar, Technische Universität München, Munich, Germany

5 Department of Neurology, Christian Doppler Medical Center, Paracelsus Medical University, Ignaz-Harrer-Str. 79, 5020 Salzburg, Austria epilepticus $(7 / 27,26 \%)$ and delirium $(4 / 27,15 \%)$. One-year survival was $82 \%$, all five deceased were male, and $3(60 \%)$ of them had "probable" disease. The non-survivors (median follow-up 1 year) were more likely to have underlying cancer and higher need for respiratory support compared to the survivors ( $p<0.041$, and $p=0.004$, respectively).

Conclusions Clinical presentations and outcomes in critically ill patients with $\mathrm{AE}$ are diverse, and the most common leading cause for intensive care unit admission was status epilepticus. The association of comorbid malignancy and the need for mechanical ventilation with mortality deserves further attention.

Keywords Autoimmune encephalitis - Intensive care unit · Mortality $\cdot$ Status epilepticus · Immune-mediated

\section{Introduction}

Encephalitis is a life-threatening medical condition of various etiologies, which affects patients of all ages and results in substantial morbidity and mortality worldwide [1,2]. Along with recent advances in diagnostic testing, autoimmune conditions have been receiving increasing recognition as causes of encephalitis [3]. Autoimmune encephalitis (AE) poses a diagnostic challenge because of its heterogeneous clinical presentations that include neurological, psychiatric, and general medical conditions. In addition, at this time antibody testing to confirm $\mathrm{AE}$ is only available at specialized centers. Direct consequences are an inconsistent coverage for antibody testing and, in many cases, delayed obtainment of results $[4,5]$. Timely diagnosis and treatment is, however, essential for favorable outcome [6-8].

Patients with $\mathrm{AE}$ often develop life-threatening complications that necessitate intensive care unit (ICU) 
admission [9, 10]. Notably, while ICU admission has been shown to be associated with poor outcome, little is known about clinical presentations and radiological and laboratory findings of those patients admitted to the ICU. In this study, we aimed to characterize underlying etiologies and the spectrum of clinical symptoms in critically ill patients with AE, their reasons for ICU admission, and evaluated which factors might be associated with death. Further, we evaluated the impact of comorbidity, the workload of nurses, and the disease severity during the first $24 \mathrm{~h}$ of ICU admission by using the Charlson's comorbidity index (CCI), the therapeutic intervention scoring system (TISS) 28, and the Simplified Acute Physiology Score (SAPS) II, respectively.

\section{Methods}

\section{Study Design}

This study was designed as a retrospective cohort study and conducted at a nine-bed neurological intensive care unit of a tertiary care university hospital. The local Ethics Committee evaluated the study protocol (Ethikkommission für das Bundesland Salzburg; 415-EP/73/534-2015). No patient consent was required due to the noninterventional design according to national regulations.

\section{Patients and Definitions}

We reviewed all medical records of consecutive adult patients with encephalitis admitted to the ICU of whom the potential AE cases were re-diagnosed and categorized the patients as having either "definite" or "probable" AE based on adapted criteria suggested by Mittal and Graus $[2,11]$. Patients in whom other acute neurological conditions were identified during the follow-up were excluded. Correspondingly, diagnostic criteria for the group "definite" were the detection of antibodies against the neuronal cell surface, synaptic, or onconeuronal protein in the cerebrospinal fluid (CSF) and/or serum. "Probable" encephalitis was diagnosed in patients, who did not fulfill the criteria for "definite" diagnosis but had at least three other supportive evidences for autoimmune CNS disease. Figure 1 provides an overview of the diagnostic criteria and the flowchart of patient selection.

\section{Data Collection}

Comorbid conditions were quantified using the Charlson's comorbidity index (CCI) [12]. Simplified Acute Physiology Score (SAPS) II was recorded as an indicator of disease severity during the first $24 \mathrm{~h}$ after ICU admission

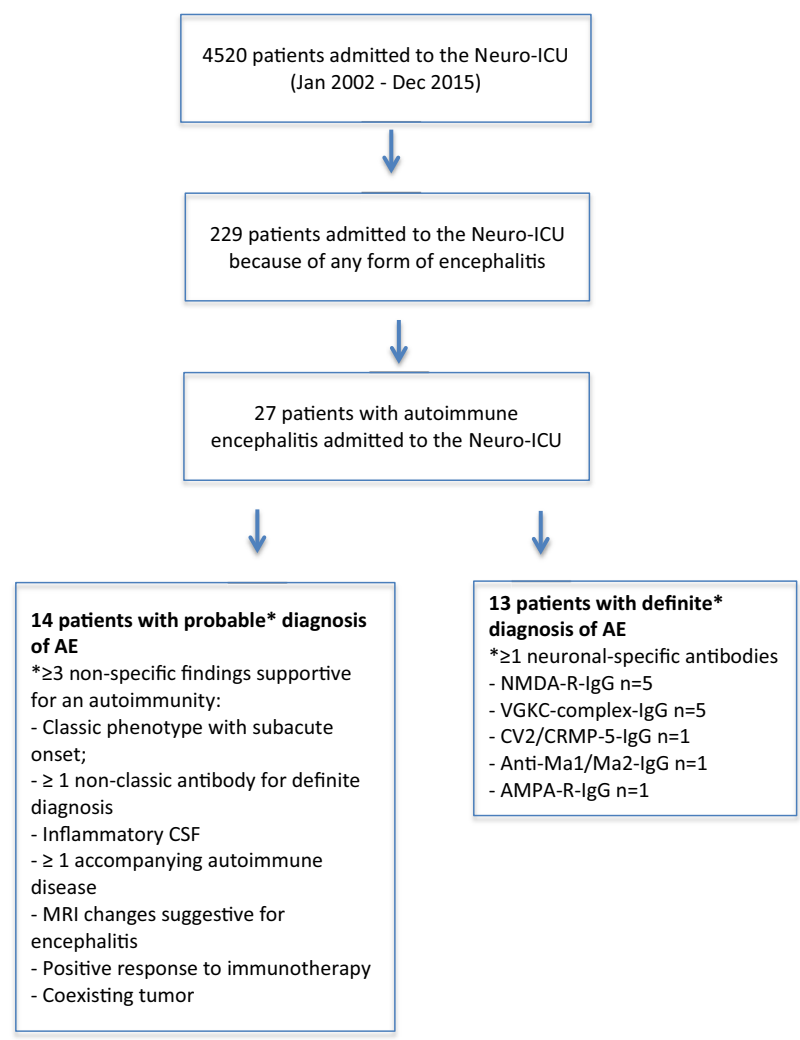

Fig. 1 Patient selection flowchart. $I C U$ intensive care unit, $A E$ autoimmune encephalitis, $C S F$ cerebrospinal fluid, MRI magnetic resonance imaging, $N M D A-R \quad N$-methyl-D-aspartate receptor, $I g G$ immunoglobulin $\mathrm{G}, V G K C$ voltage-gated potassium channel-complex, $C V 2 / C R M P 5$ collapsin response mediator protein 5, AMPA-R $\alpha-$ amino-3-hydroxy-5-methyl-4-isoxazolepropionic acid receptor

[13]. Therapeutic Intervention Scoring System (TISS) 28 was used to quantify the workload of nurses in the ICU [14]. "Definite" diagnosis of autoimmune encephalitis was based on the detection of antibodies against following antigens: Hu, Ma, Ri, Yo, Sox1, delta/notch-like epidermal growth factor-related receptor (DNER), collapsin response mediator protein 5 (CV2/CRMP5), glutamic acid decarboxylase (GAD65), dipeptidyl-peptidase-like protein-6 (DPPX), metabotropic glutamate receptor 1 (mGluR1), voltage-gated potassium channel-complex (VGKC) including leucine-rich glioma-inactivated 1 (LGI1), and contactin-associated protein-like 2 (CASPR2), $N$-methyl-Daspartate (NMDA) receptor, $\gamma$-aminobutyric acid-B $\left(\mathrm{GABA}_{\mathrm{B}}\right)$ receptor, $\alpha$-amino-3-hydroxy-5-methyl-4-isoxazolepropionic acid (AMPA) receptor, and amphyphysin. CSF was considered inflammatory if at least 2 of the following criteria were met: pleocytosis ( $\geq 5$ white cells $/ \mathrm{ml}$ ), elevated $\mathrm{IgG}$ synthesis rate, increased protein concentration ( $\geq 70 \mathrm{mg} / \mathrm{dl}$ ), and oligoclonal bands. Supportive MRI findings included mesial temporal or subcortical hyperintense changes on fluid-attenuated inversion recovery (FLAIR)/T2 imaging [1, 2]. We reviewed EEG reports and 
evaluated for the presence or absence of abnormal slow activities and epileptiform discharges. If the patient had more than one EEG, only the worst EEG was described. EEG evaluations refer to the new and validated criteria for status epilepticus $[15,16]$. Patients were screened for malignancy using sonography and fluorodeoxyflucose positron emission tomography (FDG-PET). Mortality was determined according to ICU/hospital discharge or last follow-up patient records.

\section{Statistical Analysis}

All statistical analyses were conducted using IBM SPSS version 21.0 (SPSS, Chicago, IL, USA). Descriptive methods were used to present data. Clinical, demographic, and mortality data between survivors and non-survivors (at last follow-up) were compared using the Fisher's exact or Mann-Whitney $U$ test, as appropriate. All reported $p$ values were two-tailed and considered statistically significant at $<0.05$.

\section{Results}

From January 1, 2002 until December 31, 2015, 4520 patients were treated at the NICU of the study center. Of these 229 patients were admitted because some form of encephalitis. AE was diagnosed in 27 patients $(0.6 \%)$, which comprise $67.5 \%$ of all $\mathrm{AE}$ patients diagnosed over the study period (including those not admitted to the ICU, $n=40$ in total). The temporal distribution of NICU admissions of patients with AE is presented in Fig. 2.

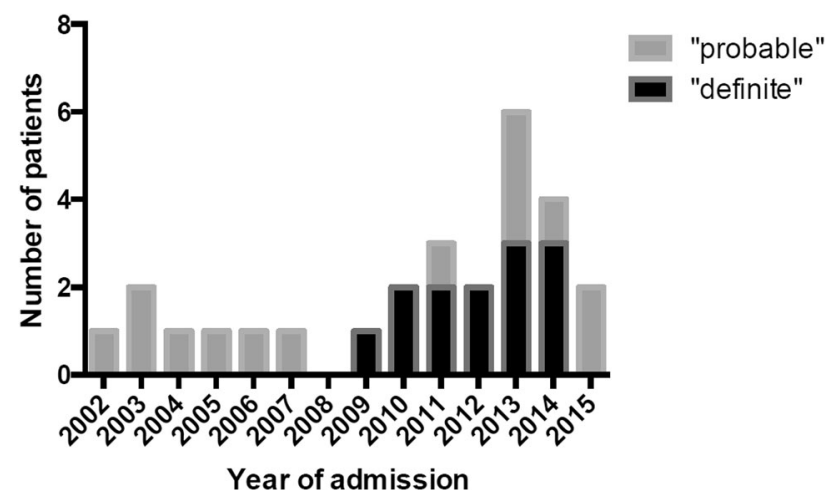

Fig. 2 Temporal distribution of neurological intensive care unit (neuro-ICU) admissions in patients with autoimmune encephalitis. Note the columns represent absolute numbers, e.g., 3 patients in each subgroup for year 2013

\section{Clinical Presentations and Characteristics}

A large proportion of $\mathrm{AE}$ patients had seizures prior to the NICU admission $(n=11,40.7 \%)$. Other conditions preceding NICU admission were personality or behavioral changes $(n=7,25.9 \%)$, subacute cognitive decline $(n=6,22.2 \%)$, headache $(n=5,18.5 \%)$, fever, and confusional state ( $n=4,14.8 \%$ each). Correspondingly, the most frequent diagnosis at the NICU among them was status epilepticus $(n=7,26 \%)$, followed by delirium and respiratory failure $(n=4,14.8 \%$ each), coma $(n=2$, $7.4 \%)$, hemiparesis, and sepsis $(n=1,3.7 \%$ each). One patient was admitted for the purpose of scheduled therapeutic plasma exchange. Demographic and clinical characteristics of critically ill patients are provided in Table 1. Further details of individual patients are shown in supplemental material for definite (e1) and probable (e2) autoimmune encephalitis.

\section{Associated Autoimmune Diseases and Tumors}

Eight patients $(29.6 \%)$ had coexisting systemic autoimmune disorders. Two patients $(7.4 \%)$, who were seropositive for anti-NMDA-R antibody, had Hashimoto's thyroiditis. The autoimmune disorders in patients with "probable" AE were the following: autoimmune thrombocytopenia, rheumatoid arthritis, systematic lupus erythematosus, vitiligo, Crohn's disease, and dermatomyositis ( $n=1,3.7 \%$ each).

Thirteen out of 27 patients $(51.8 \%)$ had the following underlying malignancies: ovarian teratoma $(n=5,38.5 \%)$, prostate $(n=2,15.4 \%)$, small cell and non-small cell lung cancer, pancreatic cancer, seminoma, CNS lymphoma, and rectal adenocarcinoma ( $n=1$ each, $7.7 \%)$. Four patients with ovarian teratoma were seropositive for anti-NMDA-R antibody, and the tumor was detected after AE diagnosis. The patient with seminoma was seropositive for anti-Ma1/ Ma2 antibody, 1 out of 2 patients with lung carcinoma was seropositive for anti-CV2/CMPV-5 antibody. The remaining patients with tumors had other suitable criteria for $\mathrm{AE}$, such as good response to immunotherapy, classical encephalitis with subacute phenotype, but without detection of specific antibodies.

One patient, who was seronegative for anti-neuronal antibodies, had first been admitted to the NICU because of limbic encephalitis in 2000, and was readmitted with hippocampal atrophy and rectal adenocarcinoma in 2013.

\section{Laboratory Findings}

Cerebrospinal fluid was examined in all but one patient. This was the patient with a scheduled therapeutic plasma exchange. 


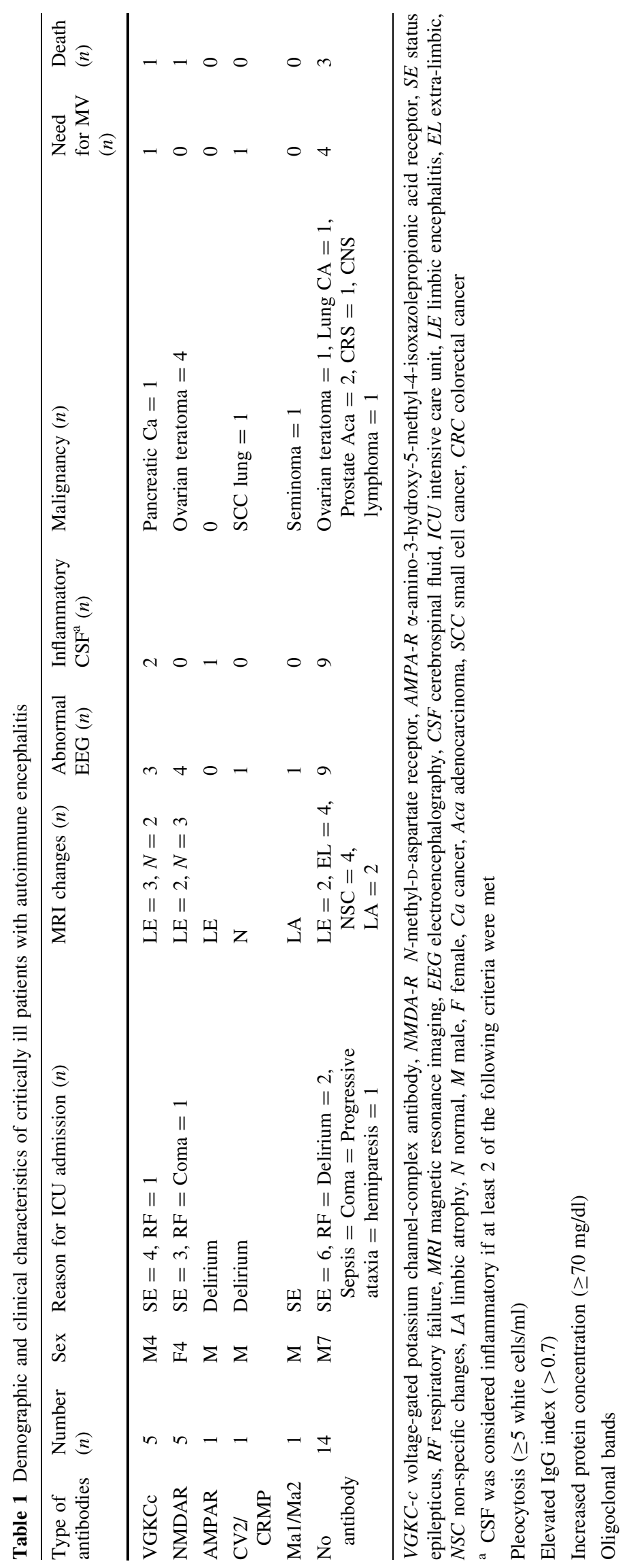


In 13 patients $(48.2 \%)$, the following anti-neuronal antibodies were detected either in serum, cerebrospinal fluid or both: anti-NMDA-R-IgG $(n=5)$, anti-VGKC-CIgG $(n=5)$, anti-Ma1/Ma2-IgG, anti-CV2/CRPM-IgG, and anti-AMPA-IgG ( $n=1$ each).

Twelve patients $(46.2 \%)$ had cerebrospinal fluid findings indicative of an inflammation (elevated $\mathrm{IgG}$ synthesis rate, high concentrations of total protein, pleocytosis, and oligoclonal bands), three of whom were categorized as having "definite" disease being additionally seropositive to VGKC-c $(n=2)$ and AMPA-R $(n=1)$ antibodies. An increased cerebrospinal fluid protein concentration $(\geq 70 \mathrm{mg} / \mathrm{dl}$ ) was detected in eleven $(91.7 \%)$ of them (median value 133, range 78-290), pleocytosis ( $\geq 5$ white cells $/ \mathrm{ml}$ ) in all twelve (median value 108.5, range 17-1048), elevated IgG synthesis rate in five (41.7\%) (median value 12.6, range 6.18-22.5), and cerebrospinal fluid-specific oligoclonal bands in two patients.

The remaining six patients did not fulfill the criteria for inflammatory CSF but had one cerebrospinal fluid abnormality: one patient had an increased protein concentration $(80 \mathrm{mg} / \mathrm{dl})$, and five patients showed pleocytosis (median value 13, range 9-35). No cerebrospinal fluid abnormalities were found in eight $(30.8 \%)$ patients.

\section{Brain Imaging}

Specific T2-signal abnormalities (hyperintensities in affected brain regions, medial temporal lobes and/or subcortical regions) were detected in 15 patients $(55.6 \%)$. These lesions were located in the limbic system in 11 patients $(40.7 \%)$ and in extra-limbic regions in four patients (14.8\%). Non-specific changes/leukoaraiosis were present in four $(14.8 \%)$, and no abnormalities in eight patients $(29.6 \%)$.

\section{EEG Findings}

An EEG (prior to ICU admission) was performed in all but one patient "who was admitted for therapeutic plasma exchange." Eighteen (69.2\%) had at least one abnormality detected in the EEG. Seven patients (2 of which were seropositive to neuronal antibodies) had EEG pattern consistent with status epilepticus. The remaining seven patients (three with "definite" diagnosis) had abnormal slow activity (generalized $n=4$, focal $n=3$ ). Extreme delta brushes were found in the EEG of a patient with antiNMDA receptor encephalitis.

\section{NICU Management, Secondary Complications}

The median length of stay in the NICU was 5 days (range 1-85). Sixteen patients $(55.6 \%)$ received immunotherapy.
These treatments included corticosteroids $(n=7)$, intravenous immunoglobulin $(n=8)$, therapeutic plasma exchange $(n=12)$, and/or rituximab $(n=2)$. Eight improved on a combination treatment. Six patients required endotracheal intubation due to respiratory failure $(n=4$, $66.7 \%$ ), refractory status epilepticus, and/or palliative stenting for pancreatic cancer ( $n=1,16.7 \%$ each). The median duration of mechanical ventilation was 14 days (range 1-27). Percutaneous dilatational tracheostomy was performed in four (14.8\%) patients, in three of them for a median duration of 12 days (range 1-19) following the endotracheal intubation, and immediately at the time of admission in one patient. The tracheostomy tube was removed after a median duration of 30.5 days (range 3-38). The summary of treatments is presented in Supplementary Material (Table_e-3).

The most common secondary complication was kidney failure $(n=4,14.8 \%)$. Aspiration pneumonia occurred in 3 patients (11.1\%). One patient developed chemotherapyinduced hepatopathy with a programmed cell death-1 (PD1) inhibitor for the treatment of non-small cell lung cancer. Further complications included urinary infection and pneumothorax, respectively.

\section{Mortality and Causes of Death}

A comparison of survivors versus non-survivors is presented in Table 2. The mortality rate at last follow-up (median 1 year, range $1-15)$ was $18.5 \%(n=5)$. One patient $(20 \%)$ died because of multi-organ failure (sepsis) in the NICU, another two (40\%) of sudden cardiac death, "one in the NICU and one after discharge in the hospital." The two remaining patients died of cardiorespiratory failure and tumor progression after hospital discharge. The survival curve of study patients is presented in Fig. 3.

\section{Discussion}

We report the spectrum of clinical symptoms, underlying etiologies and mortality among critically ill patients with AE. Our study highlights the increasing number of ICU admissions due to AE over time. This observation is most likely the result of an increased recognition of this complex disease which eventually, can lead to the fact that the disease will cease to be rare [2]. Most importantly, we found that non-survivors had a longer ICU stay, higher need for mechanical ventilation, higher comorbidity and disease severity scores, and an increased rate of underlying malignancies than survivors.

To the best of our knowledge, only one study has so far reported on the ICU management of patients with definite and probable $\mathrm{AE}$ [11]. Compared to the latter cohort with 
Table 2 Comparison of survivors versus non-survivors

\begin{tabular}{|c|c|c|c|c|}
\hline Parameter & Total $(n=27)$ & Survivors $(n=22)$ & Non-survivors $(n=5)$ & $p$ value \\
\hline Age at admission (years) & $55(24)$ & $72(23)$ & $50(29)$ & 0.006 \\
\hline Male gender $[n(\%)]$ & $16(59.3)$ & $11(50)$ & $5(100)$ & 0.054 \\
\hline Charlson's comorbidity index & $3(3)$ & $2(2)$ & $8(5.5)$ & 0.001 \\
\hline Associated tumors $[n(\%)]$ & $14(51.8)$ & $9(40.9)$ & $5(100)$ & 0.041 \\
\hline SAPS II & $25(22)$ & $20(18.3)$ & $48(30.5)$ & 0.006 \\
\hline TISS-28 & $28(6)$ & $28(6.5)$ & $29(7.5)$ & 0.8 \\
\hline Noninvasive mechanical ventilation $[n(\%)]$ & $6(22.2)$ & $2(9.1)$ & $4(80)$ & 0.004 \\
\hline Invasive mechanical ventilation $[n(\%)]$ & $4(14.8)$ & $2(91)$ & $2(40)$ & 0.1 \\
\hline Plasma exchange $[n(\%)]$ & $12(44.4)$ & $11(50)$ & $1(20)$ & 0.2 \\
\hline Vasopressor administration $[n(\%)]$ & $17(63)$ & $15(68.2)$ & $2(40)$ & 0.2 \\
\hline Length of ICU stay (days) & $5(29)$ & $5(18.5)$ & $15(46)$ & 0.04 \\
\hline
\end{tabular}

All data are given as median values with interquartile range in parentheses, unless otherwise specified

CCI Charlson's comorbidity index, SAPS simplified acute physiology score, TISS therapeutic intervention scoring system, ICU intensive care unit

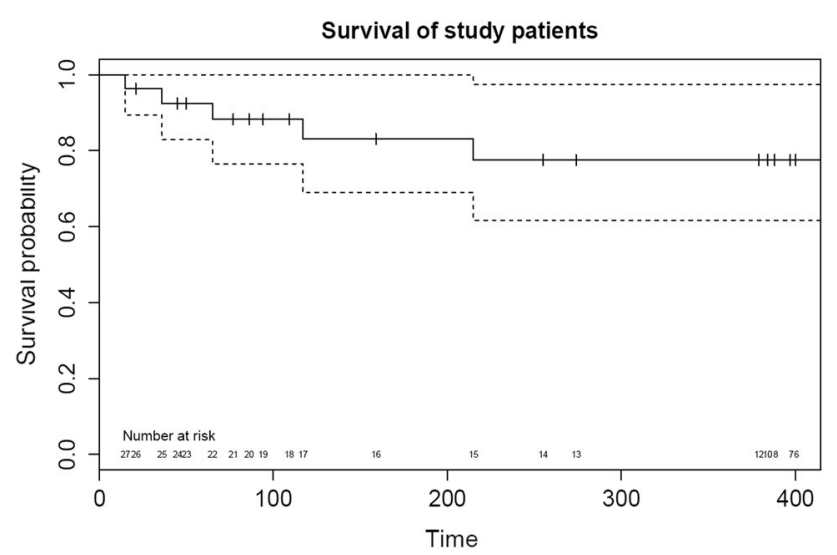

Fig. 3 Survival curve of study patients. Survival curve (solid line) with $95 \%$ confidence intervals (dotted line) with the number at risk along the $x$ axis

25 patients, our study population comprised elder patients and a higher percentage of male patients. Yet, the proportion of patients with detected anti-neuronal antibodies was similar. In accordance with the study from Mittal and colleagues, our study period covered a time when laboratory testing for many autoimmune antibodies were only scarcely available. This is likely to be the reason why the diagnosis of "definite" could only be established in about half of the cases in this cohort. Thus, the importance of the AE diagnosis based on clinical and paraclinical findings has to be appreciated, since early treatment measures are of critical importance [17].

In concert with the results from Mittal et al., we found seizures to be common (48 vs. $40.7 \%$ ) clinical manifestation in AE patients requiring ICU admission. Behavioral changes and subacute cognitive decline, in contrast, occured predominantly among seropositive AE patients in our series (38.5 vs. $7.1 \%$ ), but were more prevalent in the antibody-negative group of the mixed ICU cohort in Mittal's study (38.5 vs. $66.7 \%$, respectively). When interpreting these data, one has to take a referral bias innate to a NICU population into account.

The most common reason for ICU admission in our study population was status epilepticus (26\%). Status epilepticus is a predictor of poor outcome in general for patients with encephalitis and specifically in critically ill patients with encephalitis [18, 19]. Remarkably, only one of the study patients admitted because of status epilepticus had lethal outcome. Interestingly, a recent French study reported that the occurrence of status epilepticus during the ICU stay in patients with anti-NMDA receptor encephalitis was not associated with poor outcome [20]. For GABA $_{A}$ receptor antibody encephalitis high antibody titers were shown to be associated with a more severe clinical course and occurence of seizures, refractory status epilepticus, or both [21]. Thus, future studies should confirm and expand the relevance of antibody titers on the risk for the development of seizures.

We found that AE non-survivors (at ICU/hospital discharge/1 year) presented with more severe comorbidities, had more severe degree of critical illness as assessed by SAPS II scores, and had a higher requirement for mechanical ventilation than survivors. The latter finding is consistent with previous studies of critically ill patients suffering from encephalitis [18, 22-24]. Although not specific for an encephalitis population, comorbidity scores have been shown to be higher among neurological critical care non-survivors [25], albeit not in critically ill multiple sclerosis patients from our ICU, as we have studied previously [26]. 
As concerns the results of paraclinical investigations in our study population, only $46 \%$ showed abnormal cerebrospinal fluid findings. More than one-third of patients had MRI (FLAIR/T2) abnormalities supporting the diagnosis of AE. A recent multicenter study suggested that the cerebrospinal fluid analysis and MRI remains unremarkable in a significant proportion of patients with AE (37 and $41 \%$, respectively) [27]. Together with these reports, our results emphasize the need for a multimodal approach and a high degree of clinical suspicion to diagnose AE. Therefore, improved recognition of the disease and awareness among medical practitioners is paramount.

As described in the literature, anti-NMDA receptor encephalitis typically affects female patients prior to the age of 30 years and is frequently accompanied by ovarian teratoma [28]. Accordingly, this was also the case in four of the five study patients diagnosed with anti-NMDA receptor encephalitis in our cohort. In all except one of these patients, teratoma was subsequently detected during the hospital stay. Notably, the only patient with antiNMDA receptor encephalitis without cancer diagnosis died.

Although patients with encephalitis associated with cell surface antibodies have been shown to have a more favorable outcome compared to subjects with $\mathrm{AE}$ and intracellular antibodies [29], both deceased patients with "definite" autoimmune diagnosis in our series had antineuronal antibodies to cell surface. In both cases, death occurred only after hospital discharge.

Important limitations need to be considered when analyzing the results of our study. First, the retrospective study design provides lower diagnostic accuracy, and the relatively small sample size limits the generalizability of our findings. However, our results were comparable to the aforementioned series in most aspects [11]. Secondly, we acknowledge that the number of AE patients with specific neuronal antibodies might be underrepresented as a systematic retesting, and re-evaluation of both serum and CSF was inconsistent [30]. Moreover, patients with intracellular antigens may be low for the reason of management by oncologists. Finally, our study did not include patients younger than 18 years, while in one patient there were anamnestic hints pointing at earlier onset of AE. Therefore, we might have missed $\mathrm{AE}$ subtypes that more frequently occur in children. Concededly, studies with larger catchment area including other (medical, pediatric, etc.) ICU facilities would further complement our findings.

Our study suggests that clinical presentations and reasons for intensive care admission in critically ill $\mathrm{AE}$ patients are diverse, and the underlying conditions are dominated by status epilepticus. The association of comorbidity, malignancy, and the need for mechanical ventilation with adverse outcome should be considered in clinical practice and deserves further investigation in prospective studies. Further studies should also take the functional and cognitive status as outcome measure into account.

Acknowledgements Open access funding provided by Paracelsus Medical University.

\section{Compliance with Ethical Standards}

Conflict of interest All authors declare that they have no conflict of interest.

Open Access This article is distributed under the terms of the Creative Commons Attribution 4.0 International License (http:// creativecommons.org/licenses/by/4.0/), which permits unrestricted use, distribution, and reproduction in any medium, provided you give appropriate credit to the original author(s) and the source, provide a link to the Creative Commons license, and indicate if changes were made.

\section{References}

1. Venkatesan A, Tunkel AR, Bloch KC, et al. Case definitions, diagnostic algorithms, and priorities in encephalitis: consensus statement of the international encephalitis consortium. Clin Infect Dis. 2013;57:1114-28.

2. Graus F, Titulaer MJ, Balu R, et al. A clinical approach to diagnosis of autoimmune encephalitis. Lancet Neurol. 2016;15: 391-404.

3. Dalmau J, Gleichman AJ, Hughes EG, et al. Anti-NMDA-receptor encephalitis: case series and analysis of the effects of antibodies. Lancet Neurol. 2008;7:1091-8.

4. Antoine JC. Autoimmune encephalitis: paving the way for early diagnosis. Lancet Neurol. 2016;15:349-50.

5. Dubey D, Sawhney A, Greenberg B, et al. Characteristics of autoimmune encephalitis at two teaching hospitals. Neurology. 2015;84:P4.058.

6. Ramanathan S, Mohammad SS, Brilot F, et al. Autoimmune encephalitis: recent updates and emerging challenges. J Clin Neurosci. 2014;21:722-30.

7. Gaieski DF, Nathan BR, O'Brien NF. Emergency neurologic life support: meningitis and encephalitis. Neurocrit Care. 2015; 23(Suppl 2):S110-8.

8. Brophy GM, Human T, Shutter L. Emergency neurological life support: pharmacotherapy. Neurocrit Care. 2015;23(Suppl 2): S48-68.

9. Titulaer MJ, McCracken L, Gabilondo I, et al. Treatment and prognostic factors for long-term outcome in patients with antiNMDA receptor encephalitis: an observational cohort study. Lancet Neurol. 2013;12:157-65.

10. Claassen J, Riviello JJ Jr, Silbergleit R. Emergency neurological life support: status epilepticus. Neurocrit Care. 2015;23(Suppl 2):S136-42.

11. Mittal MK, Rabinstein AA, Hocker SE, et al. Autoimmune encephalitis in the ICU: analysis of phenotypes, serologic findings, and outcomes. Neurocrit Care. 2016;24:240-50.

12. Cleves MA, Sanchez N, Draheim M. Evaluation of two competing methods for calculating Charlson's comorbidity index when analyzing short-term mortality using administrative data. J Clin Epidemiol. 1997;50:903-8. 
13. Le Gall JR, Lemeshow S, Saulnier F. A new simplified acute physiology score (saps Ii) based on a European/North American multicenter study. JAMA. 1993;270:2957-63.

14. Miranda DR, de Rijk A, Schaufeli W. Simplified therapeutic intervention scoring system: the Tiss-28 items-results from a multicenter study. Crit Care Med. 1996;24:64-73.

15. Leitinger M, Beniczky S, Rohracher A, et al. Salzburg consensus criteria for non-convulsive status epilepticus-approach to clinical application. Epilepsy Behav. 2015;49:158-63.

16. Trinka E, Cock H, Hesdorffer D, et al. A definition and classification of status epilepticus-report of the Ilae task force on classification of status epilepticus. Epilepsia. 2015;56:1515-23.

17. Nosadini M, Mohammad SS, Ramanathan S, et al. Immune therapy in autoimmune encephalitis: a systematic review. Expert Rev Neurother. 2015;15:1391-419.

18. Thakur KT, Motta M, Asemota AO, et al. Predictors of outcome in acute encephalitis. Neurology. 2013;81:793-800.

19. Venkatesan A. Epidemiology and outcomes of acute encephalitis. Curr Opin Neurol. 2015;28:277-82.

20. de Montmollin E, Demeret S, Brule N, et al. Anti- $N$-methyl-Daspartate receptor encephalitis in adult patients requiring intensive care. Am J Respir Crit Care Med. 2016. doi:10.1164/rccm. 201603-0507OC.

21. Petit-Pedrol M, Armangue T, Peng X, et al. Encephalitis with refractory seizures, status epilepticus, and antibodies to the GABAA receptor: a case series, characterisation of the antigen, and analysis of the effects of antibodies. Lancet Neurol. 2014;13:276-86.
22. Leroy G, Devos P, Lambiotte F, et al. One-year mortality in patients requiring prolonged mechanical ventilation: multicenter evaluation of the provent score. Crit Care. 2014;18:R155.

23. Hough CL, Caldwell ES, Cox CE, et al. Development and validation of a mortality prediction model for patients receiving 14 days of mechanical ventilation. Crit Care Med. 2015;43:2339-45.

24. Sonneville R, Gault N, de Montmollin E, et al. Clinical spectrum and outcomes of patients with encephalitis requiring intensive care. Eur J Neurol. 2015;22(6-16):e11.

25. Quan H, Li B, Couris CM, et al. Updating and validating the charlson comorbidity index and score for risk adjustment in hospital discharge abstracts using data from 6 countries. Am J Epidemiol. 2011;173:676-82.

26. Karamyan A, Dunser MW, Wiebe DJ, et al. Critical illness in patients with multiple sclerosis: a matched case-control study. PLoS ONE. 2016;11:e0155795.

27. Dubey D, Sawhney A, Greenberg B, et al. The spectrum of autoimmune encephalopathies. J Neuroimmunol. 2015;287:93-7.

28. Acien P, Acien M, Ruiz-Macia E, et al. Ovarian teratoma-associated anti-NMDAR encephalitis: a systematic review of reported cases. Orphanet J Rare Dis. 2014;9:157.

29. Zekeridou A, Rossetti AO, Hottinger AF, et al. Anti-neuronal antibodies: a rapidly developing field. Rev Med Suisse. 2013;9:909-14.

30. Giometto B, Grisold W, Vitaliani R, et al. Paraneoplastic neurologic syndrome in the PNS Euronetwork database: a european study from 20 centers. Arch Neurol. 2010;67:330-5. 